\title{
A Novel Approach to Analog Signal Isolation through Digital Opto-coupler (YOUTAB)
}

\author{
Mahdi KhosroAnjom (Corresponding author) \\ MAPNA Electrical and Control Engineering \& Manufacturing Co. (MECO) \\ P.O.Box 31585-1551, Karaj, Iran \\ Tel: 98-912-526-0239 E-mail: mahdi.anjom@gmail.com \\ Sajad Moslehi \\ MAPNA Electrical and Control Engineering \& Manufacturing Co. (MECO) \\ P.O.Box 31585-1551, Karaj, Iran
}

Tel: 98-912-362-9041Ｅ-mail: moslehi.sd@gmail.com

Received: February 19, 2011

Accepted: April 11, 2011

doi:10.5539/mas.v5n3p225

\begin{abstract}
Major concerns which clearly express necessity of analog isolator applications, are removing ground loop, increasing common-mode voltage rejection, improving noise immunity and protecting operator, expensive equipment and data from transient voltages. Optical coupling is an approach to signal isolation in which all mentioned benefits are covered. This paper is going to propose a novel idea of a particular optical analog signal isolator. It is introduced for the first time and therefore the authors named it YOUTAB. The digital photocoupler NEC-PS2501 constitutes the main structure of this new isolator. In general, the proposed configuration consist of two different parts that one of them transfers the signal and the other establishes a negative feedback to ensure that the output signal has the same features of the input signal. The operation, analysis and computer simulation results are shown. The simulation results show that this novel proposed analog isolator is linear in a wide range of inputs.
\end{abstract}

Keywords: Analog isolation, Optocoupler, Optical coupling, Negative feedback, Embedded systems, YOUTAB

\section{Introduction}

The application of galvanic insulators has continuously increased in electronic circuits in order to achieve a high safety and reliability in signal process and transmission circuits. Considerable evidences do exist that such features are highly desired through obliged standards and/or special requirements like complex high-speed electronic systems on aircraft and space vehicles.

For many years, ground isolation has been a serious challenge and important aspect of electronics design. Frequently, for designers, it is essential to transmit some signals between separated subsystems associated with different grounding systems. Perhaps, a considerable problem here is the high differential potential between two different grounds which could lead to damage of devices while human safety is lost. Other phenomenon such ground loops in many applications caused to considerable decline in signal quality. In practical cases, galvanic isolation is used for elimination of these kinds of interferences. Among the isolation schemes the optical isolators are highly implemented specially in small signal analog circuits.

A whole raft of ideas and strategies for optical isolation has been studied in many papers but what is going to be proposed here, is a technical novel idea of analog signal isolation using optocouplers. The authors have nominated this configuration as YOUTAB.

In ordinary solution, the analog signals are converted to digital and then are transmitted via an optocouplers but in the newer methods, analog signals are transmitted directly and without conversion. In YOUTAB configuration, not only the analog signal is transmitted directly but also, the passed on current sample is used to establish a negative feedback [Figure 1] for achieving to a linear signal transmitting via internal close loop. Therefore this feature distinguishes our idea from existing analog isolators.

A novel approach of analog signal isolation is proposed. First, some similar solutions are described to show what 
other methods are used in terms of analog isolation. Then, in the next two sections, the concerns which exist in the area of analog isolation and the solution of them are presented respectively. After that YOUTAB modeling section describes the fundamental concept of YOUTAB and its arrangement. Consequently Analysis section, discusses about basic relations of YOUTAB and provides major equations. Lastly, the simulation results explain different aspects of YOUTAB operation.

\section{Related Works}

In recent years, various techniques and configurations have been introduced and studied for analog signals isolation. Modulation-Demodulation method is occasionally used for this kind of galvanic isolation. In this complex and multipart solution analog signal is modulated and then transmitted through an optocouplers and finally the basic signal is demodulated. The other approach is basically as same as former one but it utilizes A/D and $\mathrm{D} / \mathrm{A}$ converters instead of modulation technique. However, in addition to those mentioned drawbacks here the A/D conversion time conversion may assume as a considerable limitation too. Next solution uses a LED as input element, and output element is always a photoconductive cell. The resistance of the photocell depends on the amount of light falling on the cell. For a given illumination, the amount of electrical current through the cell depends on the voltage applied. Although it benefits from a simple construction and low cost characteristic, the slow response time caused to restriction of use in high-speed applications. Between the emerged methods for optical analog isolators, the IL300 isolators have good features of a rather wide bandwidth and high linearity but the concept does not include of internal feedback and this must to be occurs via an external circuit.

\section{Problem Statement}

All above approaches have some limitations which could classify in two categories. Firstly, the lack of technological capability of implemented devices may not lead to desired response. Indeed, the root of this kind of difficulties lies in nature of construction and has no solution. For instance, in photocell the pace of change in resistance is very slow that is in account of its innate features. In the second category, the problems are irrelevant to fundamentals however, solving them needs to afford high costs and add peripheral circuits.

In signal conversion method, achieving to a high quality isolator extremely depends on the used Analog to Digital Converter speed and sampling time. To provide this sort of features, in some cases, caused to significant extra cost.

\section{Solution}

One of the approaches used in analog isolators is optical isolation method. In this method the media for transmitting the signal is light and the physical isolation barrier is typically an air gap. YOUTAB configuration introduces a new analog isolator that works based on optical coupling method. It consists of two major parts that each part contains a digital optocoupler. The implemented type of optocouplers in YOUTAB contains Light Emitting Diode at input side and a phototransistor at output.

The transmission media is mutually built through the digital optocouplers. They are electrically connected together but contribute in such way that the output signal is isolated from input. The block diagram of YOUTAB is presented in Figure 2. Block A, the isolator part, passes the input signal on to output while block B is used to establish a negative feedback.

\section{Modeling}

In electronic circuits, an optocoupler schematic diagram is illustrated with combination of its internal elements. Figure $3 \mathrm{~A}$ shows the common schematic of a standard phototransistor optocoupler. Figure $3 \mathrm{~B}$ is an expanded schematic that include a collector-base photo-detector.

The input LED current, $\mathrm{I}_{\mathrm{i}}$, creates an optical flux, which is detected by the photodiode and caused to develop a photocurrent, $\mathrm{I}_{\mathrm{cb}}$. This current is amplified by the transistor as collector-emitter current, Ice. The current gain of the device is defined as a current transfer ratio (CTR):

$$
C T R=\frac{I_{c e}}{I_{i}} \times 100 \%
$$

The relationship given in equation 1 can be shown in Figure 4. In this circuit Ice is produced by a controlled current source. This model particularly may used in optocouplers which works in active region.

To introduce a more precise model of the YOUTAB, The optocoupler model illustrated with Figure 4 have to be implement in Figure 2. This model might be used for extracting the relevant equations and circuit analyzing of the YOUTAB (Figure 5). 


\section{Analysis}

To analyze the proposed model of YOUTAB, fundamental electrical circuit laws are used. Utilizing Figure 5, it can be seen that the Input signal (Is) is divided in two part Ii and If. An optocoupler in circuit A transmits the current Ii with CTRA factor as Io (equation 2) which is shown by a dependant current source in circuit A.

$$
I_{o}=C T R_{A} \cdot I_{i}
$$

From the optocouplers characteristics (Figure 6) it is obvious that the CTR in optocouplers is affected by several parameters such as manufacturing technology, current-gain of the transistor and LED current. The malfunction of an optocoupler probably is distortion on output signal. Since here, the operation of signal isolation has not been completed although the signal has transmitted to the output.

Generally, a negative feedback could establish a stable gain to resolve this problem. In YOUTAB this feedback is implemented through circuit B. The current Io appears as If at input and related equation is:

$$
I_{f}=C T R_{B} \cdot I_{o}
$$

The expected performance of this feedback is to maintain a constant total current transfer ratio with below definition:

$$
C T R_{Y}=\frac{I_{o}}{I_{s}}
$$

This feedback, in addition to linearization, makes YOUTAB independent from other unwanted parameters which cause any kind of distortions.

The transfer function of YOUTAB helps to examine its characteristics. This is a relation between input and output. Total input current of the circuit A can be gained from difference of the Is and If:

$$
I_{i}=I_{S}-I_{f}
$$

Substitute from (5) and (3) in (2) Io can be calculated as:

$$
I_{o}=C T R_{A}\left(I_{S}-C T R_{B} I_{o}\right)
$$

Solving the equation 6 for Io results:

$$
I_{o}=\frac{I_{S} \cdot C T R_{A}}{1+C T R_{A} \cdot C T R_{B}}
$$

As a result the total gain will be:

$$
C T R_{Y}=\frac{I_{o}}{I_{S}}=\frac{C T R_{A}}{1+C T R_{A} \cdot C T R_{B}}
$$

As circuit A and B use the same optocoupler and YOUTAB operate in their linear part of current transfer ration the total gain CTRY remain constant.

\section{Simulation and Experimentation}

The Integrated Circuit Emphasis (SPICE) PSpice program is used to simulate proposed configuration in Figure 5. The digital optocoupler NEC-PS2501 was used and to ensure result reliability no peripheral circuit was used.

In Figure 7 the amount of output current has been depicted respected to input current (Is). In simulation of YOUTAB, input current has been increased from $0 \mathrm{~mA}$ to $20 \mathrm{~mA}$ by equal steps of $0.1 \mathrm{~mA}$. In the meanwhile, output current has roused from the same current in the input and $\mathrm{I}_{\mathrm{o}}$ hits a high of $5.7 \mathrm{~mA}$ in final step. Obviously, this transfer function looks linear in most of working points, however in some small areas at the start of the graph and at the end of it bending is observed. In this simulation the reliable range of linear operation for YOUTAB is observable between 4-16mA, which may conditioned and improved with external circuits. To be sure an AC signal of 4-16mA, F=1 KHz applied to YOUTAB and resulted signal has been illustrated in Figure 8 .

\section{Conclusion}

The proposed configuration of the isolator (YOUTAB) introduces a new approach of galvanic isolation for analog signals. As the analysis and simulation result show, this method provides the possibility of contribution for the transmitted signal into an isolated negative feedback by which the isolator becomes independent from distortions and widely linear. Other solutions conceptually use indirect sample of the output for feedback control. 
The simplicity of YOUTAB makes it useful to be used in the embedded systems.

\section{References}

A. J. Peyton, V. Walsh. (1996). Analog electronics with Op Amps(2nd edition), page, 27, Cambridge University Press.

Alessandria, A.S. \& La Magna, L. \& Renna, M.C. \& Fragapane, L. \& Coffa, S. (2002). Integrated Si-based Opto-Couplers: a Novel Approach to Galvanic Isolation, IEEE Solid-State Device Research Conference, Proceeding of the 32nd European, DOI: 10.1109/ESSDERC.2002.195014

Analog Devices Inc. (2000). 1B22, Programmable Isolated Voltage-to-Current Converter. [Online] Available: www.analog.com/static/imported-files/data_sheets/1B22.pdf

Burr-Brown Corporation. (1997). ISO807, Isolated 16-Bit Sampling ANALOG-TO-DIGITAL CONVERTER. [Online] Available: www.datasheetcatalog.org/datasheet/BurrBrown/mXvvuyy.pdf

Camin, D.V. \& Pessina, G. (2000). Differential optocoupler amplifier with low noise, low power and balanced output, Nuclear Science, IEEE Transactions, Volume 47, Issue 6, DOI: 10.1109/23.903843

EG\&G Optoelectronics. (1997). VTL5C1-5C2, Analog Optical Isolators. [Online] Available: http://www.aikenamps.com/VTL5C1.pdf

Elya B. Joffe \& Kai-Sang Lock. (2010). GROUNDS FOR GROUNDING A Circuit-to-System Handbook, chapter 3, A JOHN WILEY \& SONS, INC. PUBLICATION

Frank Tridge. (2007). Analog electronics with Op Amps (4th edition), page, 537, springer science+business media

G. Vijayaraghavan \& Mark Brown \& Malcolm Barnes. (2004). Practical Grounding, Bonding, Shielding and Surge Protection, chapter 8 , Newnes

James William Nilsson \& Susan A. Riedel. (2005). Introduction to PSpice manual, using ORCad release 9.2 to accompany Electric circuits, Volume 1, Pearson/Prentice Hall

NEC Corporation, PS2501-1, Photocoupler. [Online] Available: http://datasheet.octopart.com/PS2501-1-NEC-datasheet-101499.pdf

R. D. Schrimpf \& D. M. Fleetwood. (2004). Selected Topics in Electronicsand Systems, Radiation effects and soft errors in integrated circuits and electronic devices, Vol. 34, page 409, World Scientific publishing Co.

Reed, R.A. \& Marshall, P.W. \& Johnston, A.H. \& Barth, J.L. \& Marshall, C.J. \& LaBel, K.A \& D'Ordine, M. \& Kim, H.S. \& Carts, M.A. (1998). Emerging optocoupler issues with energetic particle-induced transients and permanent radiation degradation, IEEE Transactions on Nuclear Science, Volume: 45, Issue: 6

Siemens, IL300 LINEAR OPTOCOUPLER, [Online] Available http://www.datasheetcatalog.org/datasheet/siemens/IL300.pdf

Simoes, J.B. \& Silva, R.M.C. \& Morgado, A.M.L.S. \& Correia, C.M. (1996). The Optical Coupling of Analog Signals, IEEE Transactions on Nuclear Science, Vol. 43, Issue 3, DOI: 10.1109/23.507168
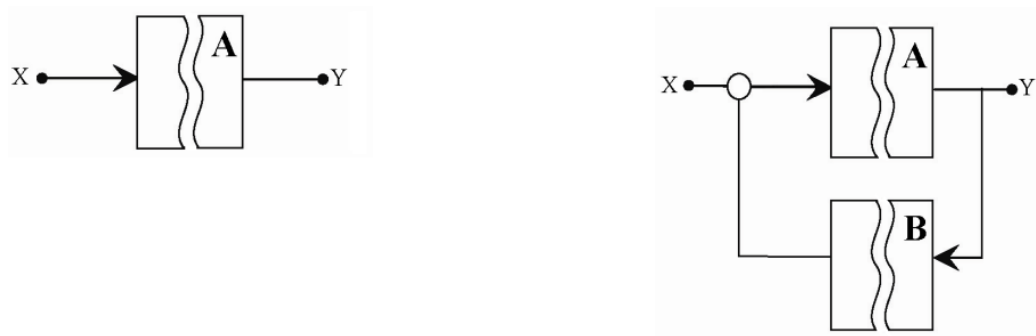

Figure 1. (a) ordinary Isolation without feedback (b) Isolation with negative feedback (YOUTAB) 


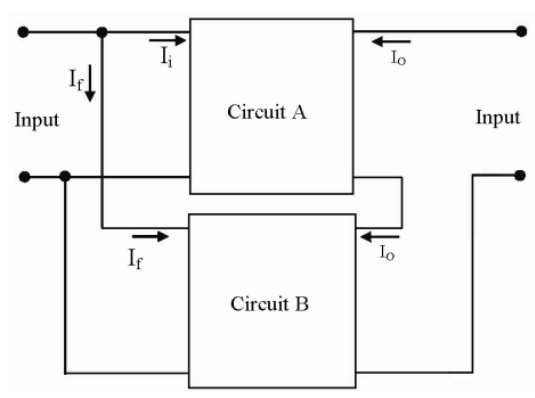

Figure 2. block diagram of YOUTAB

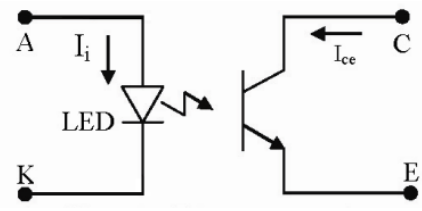

A. Simple Phototransistor

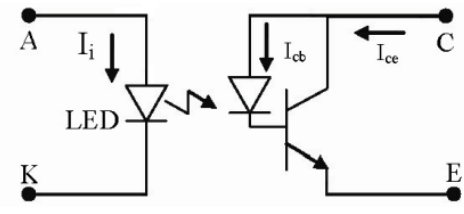

B. Expanded Simple Phototransistor

Figure 3. optocoupler schematic diagram

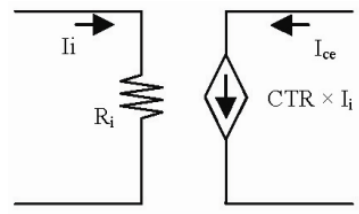

Figure 4. optocoupler model

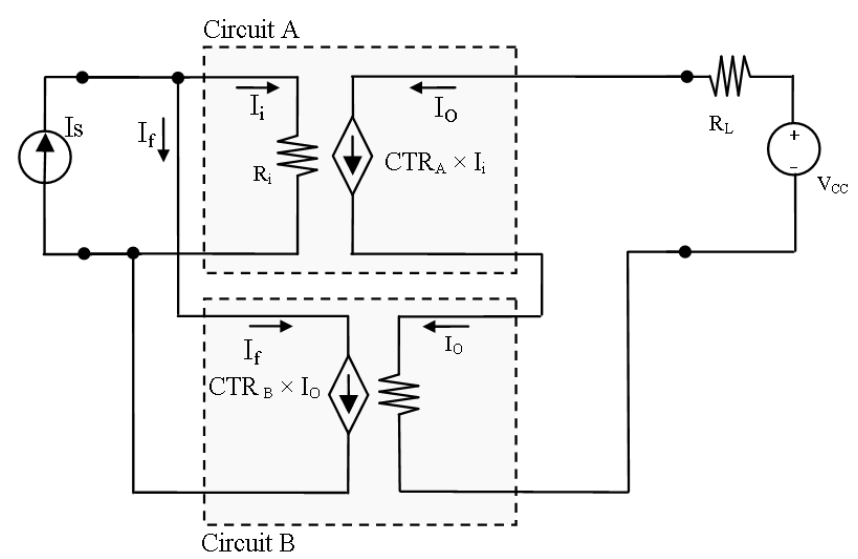

Figure 5. YOUTAB model 


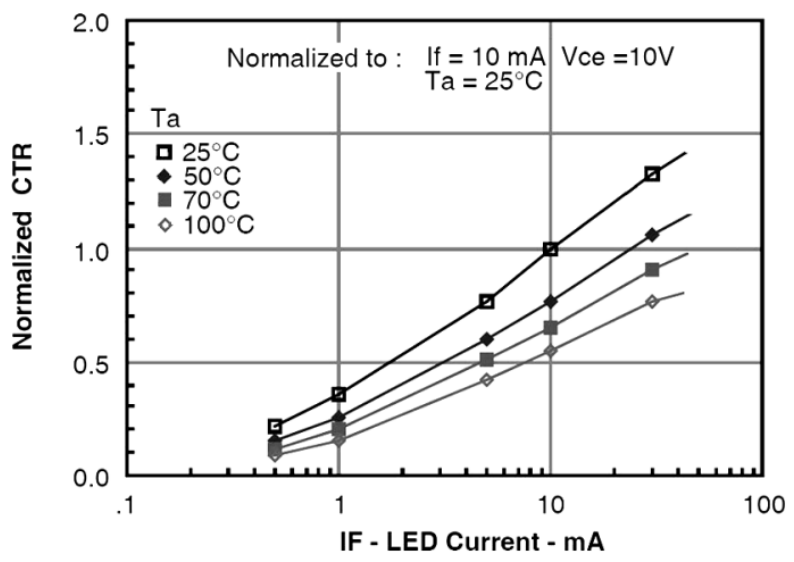

Figure 6. Current Transfer Ratio vs. Forward current

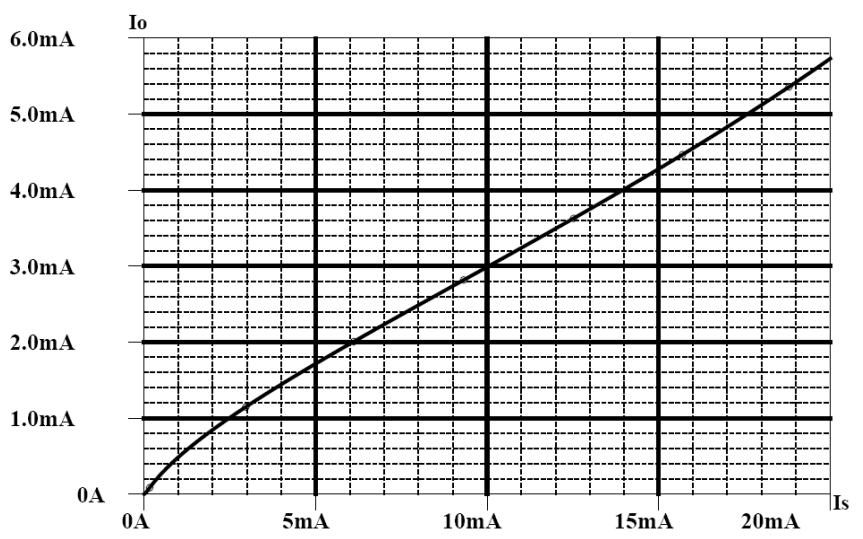

Figure 7. Output current vs. Input Current

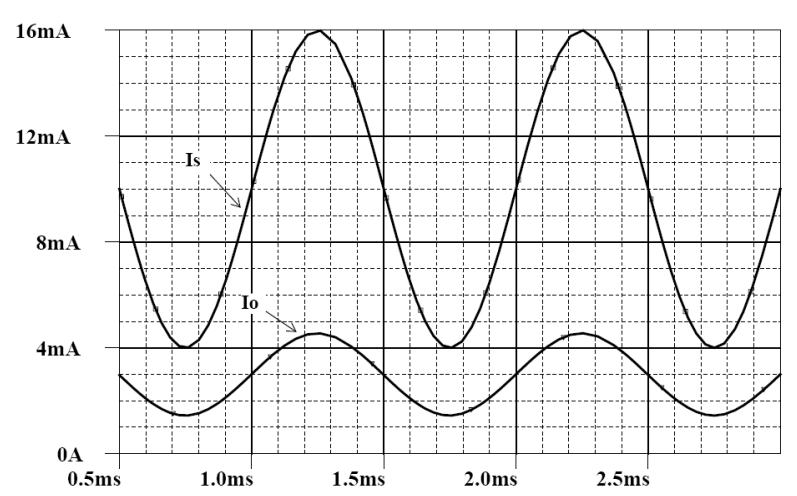

Figure 8. Sample response of YOUTAB to AC signal (4-16mA, F=1 KHz) 